\title{
Generation of coupled global and local magnetic fields by a cellular MHD dynamo
}

\author{
A. V. Getling ${ }^{1} \dagger$, R. D. Simitev ${ }^{2}$ and F. H. Busse ${ }^{3}$ \\ ${ }^{1}$ Institute of Nuclear Physics, Lomonosov Moscow State University, 119992 Moscow, Russia \\ email: A.Getling@ru.net \\ ${ }^{2}$ Department of Mathematics, University of Glasgow, G12 8QW Glasgow, UK \\ email: rs@maths.gla.ac.uk \\ ${ }^{3}$ Institute of Physics, University of Bayreuth, D-95440 Bayreuth, Germany \\ email: Friedrich.Busse@uni-bayreuth.de
}

\begin{abstract}
The convection-driven MHD dynamo in a rotating spherical shell is simulated numerically. Convection cells are regarded as a connecting link between the global and local electromagnetic processes. Local (in many cases, bipolar) magnetic structures are regularly produced by convection cells. Dynamo regimes in "thick" and "thin" shells are discussed. In the first case, the "general" magnetic field maintained by the dynamo has a sign-alternating dipolar component, which varies cyclically, although not periodically. The local structures, as they disintegrate, change into background fields, which drift toward the poles. From time to time, reversals of the magnetic fields in the polar regions occur, as "new" background fields expel the "old" fields. In the second case, the system settles down to a nearly stationary regime without polarity reversals.
\end{abstract}

Keywords. magnetic fields, convection, MHD

\section{Introduction}

Mean-field electrodynamics can satisfactorily describe the behaviour of large-scale magnetic fields of cosmic bodies, including polarity reversals and the propagation of dynamo waves. However, such problems as the formation of local magnetic fields and their relationship to the global fields completely fall beyond the scope of mean-field theories. To obtain a unified description of both the global and local processes, we need "deterministic" models that deal with structural elements of the velocity field and magnetic field instead of the averaged parameters of the turbulent flow.

The idea that convection cells in the solar subphotospheric zone could be a connecting link between global and local magnetic fields traces back to the mid-1960s. Tverskoy (1966), based on a simple kinematic model, has shown that a flow with the topology of a convection cell can amplify the magnetic field and produce bipolar magnetic configurations. This approach was also used by Getling \& Tverskoy (1971a, 1971b) to construct a model of the global MHD dynamo in a rotating spherical shell, whose building blocks are convection cells locally amplifying the magnetic field.

In recent years, the above property of the convection cells was also demonstrated in numerical simulations by Getling (2001) for incompressible and by Dobler \& Getling (2004) for compressible fluids. As for the simulation of the dynamo process in stellar convection zones on both global and local scales, the currently available computing resources cannot ensure an adequate description of the whole range of scales of convective

$\dagger$ The work of A.V.G. was supported by the Deutscher Akademischer Austauschdienst, European Graduate College "Non-Equilibrium Phenomena and Phase Transitions in Complex Systems," and Russian Foundation for Basic Research (project code 04-02-16580). 
motions. However, reproducing convection cells of a specific characteristic size in a global numerical model is quite possible.

Here, we numerically simulate the dynamo action of cellular magnetoconvection in a rotating spherical shell. The bipolar local magnetic configurations produced by convection cells can in principle be associated with local manifestation of solar activity, and their subsequent evolution can lead to the regeneration of the global poloidal field.

\section{Formulation of the problem and numerical technique}

We consider a spherical shell of thickness $d=r_{\mathrm{o}}-r_{\mathrm{i}}$ (where $r_{\mathrm{o}}$ and $r_{\mathrm{i}}$ are the outer and inner radii of the shell; $\eta=r_{\mathrm{i}} / r_{\mathrm{o}}$ is specified as a geometrical parameter of the problem), which is filled with an electrically conducting fluid and rotates at a constant angular velocity $\boldsymbol{\Omega}$ about a fixed axis $\hat{\mathbf{e}}_{z}$. We use a Boussinesq approximation with a small quadratic term included in the temperature dependence of density and assume that internal heat sources are uniformly distributed throughout the shell with a mass density $q$. Both the quadratic term and internal heating favour the development of polygonal convection cells similar to the cells observed on the Sun (instead of meridionally stretched, banana-like convection rolls).

Under static conditions, in the absence of fluid motion, the heat-transfer equation yields the following temperature distribution (from here on, we measure the radial coordinate $r$ in units of the shell thickness $d)$ :

$$
T_{\mathrm{S}}=\beta_{0}-\frac{\beta}{2} d^{2} r^{2}+\frac{\beta_{1}}{d} \frac{1}{r}, \quad \beta=\frac{q}{3 \chi c_{p}}, \quad \beta_{1}=\frac{\eta d \Delta T}{(1-\eta)^{2}},
$$

where $\chi$ is the thermal diffusivity, $c_{p}$ is the specific heat at constant pressure, and $\beta_{0}$ is a constant. Generally, $\Delta T$ is related to the actual $T_{\mathrm{i}}-T_{\mathrm{o}}$ as follows:

$$
\Delta T=T_{\mathrm{i}}-T_{\mathrm{o}}-\frac{1}{2} \beta d^{2} \frac{1+\eta}{1-\eta}
$$

it reduces to $T_{\mathrm{i}}-T_{\mathrm{o}}$ in the case of $q=0$. The shell is self-gravitating, and the gravitational acceleration averaged over a spherical surface $r=$ const can be written as $\mathbf{g}=-(\gamma d) \mathbf{r}$, where $\mathbf{r}$ is the position vector with respect to the centre of the sphere and $r$ is its length measured, as specified above, in units of $d$. In addition to $d$, the time $d^{2} / \nu$, the temperature $\nu^{2} / \gamma \alpha d^{4}$ (where $\alpha$ is the volumetric coefficient of thermal expansion), and the magnetic induction $\nu(\mu \rho)^{1 / 2} / d$ are used as scales for the dimensionless description of the problem, where $\nu$ denotes the kinematic viscosity of the fluid, $\rho$ is its density, and $\mu$ is its magnetic permeability. We denote the magnetic diffusivity as $\nu_{\mathrm{m}}$.

The physical parameters of the problem are two Rayleigh numbers [specified by the internal heat sources $q$ and by the externally specified temperature difference $T_{\mathrm{i}}-T_{\mathrm{o}}$; see (2.2)], the Coriolis number, and the hydrodynamic and magnetic Prandtl numbers:

$$
R_{\mathrm{i}}=\frac{\alpha \gamma \beta d^{6}}{\nu \chi}, \quad R_{\mathrm{e}}=\frac{\alpha \gamma \Delta T d^{4}}{\nu \chi}, \quad \tau=\frac{2 \Omega d^{2}}{\nu}, \quad P=\frac{\nu}{\chi}, \quad P_{\mathrm{m}}=\frac{\nu}{\nu_{\mathrm{m}}} .
$$

Since the velocity field $\mathbf{u}$ as well as the magnetic induction $\mathbf{B}$ are solenoidal vector fields, they can be represented in terms of poloidal and toroidal components as

$$
\mathbf{u}=\nabla \times(\nabla v \times \mathbf{r})+\nabla w \times \mathbf{r}, \quad \mathbf{B}=\nabla \times(\nabla h \times \mathbf{r})+\nabla g \times \mathbf{r} .
$$

We assume stress-free boundaries with fixed temperatures. For the magnetic field, electrically insulating boundaries are used, so that the poloidal component of the field inside the shell must be matched to a potential fields outside the shell. 


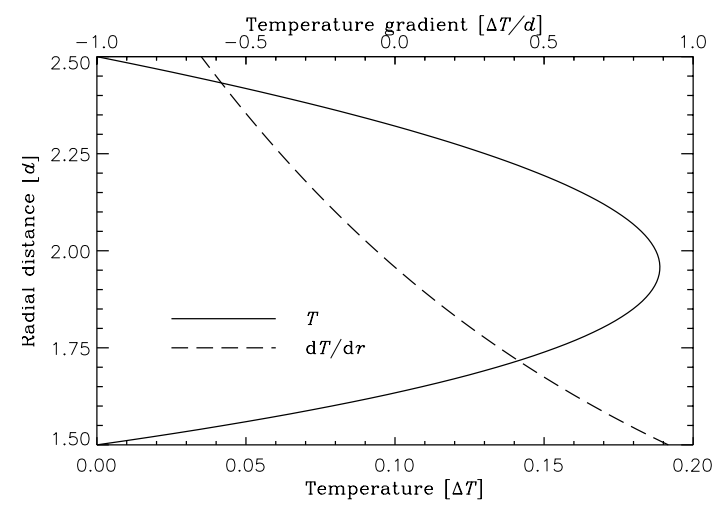

Figure 1. Static profiles of the temperature and temperature gradient in the thick-shell model with internal heating.

We use the pseudospectral method described by Tilgner \& Busse (1997) and Tilgner (1999), which is based on an expansion of all dependent variables in spherical harmonics for the $\theta$ and $\varphi$ dependences; in particular, for the magnetic scalars,

$$
g=\frac{1}{r} \sum_{l=0}^{\infty} \sum_{m=-l}^{l} G_{l}^{m}(r, t) P_{l}^{m}(\theta) \exp \{i m \varphi\}, h=\frac{1}{r} \sum_{l=0}^{\infty} \sum_{m=-l}^{l} H_{l}^{m}(r, t) P_{l}^{m}(\theta) \exp \{i m \varphi\}
$$

(with truncating the series at an appropriate upper bound for $l$ ), where $P_{l}^{m}$ denotes the associated Legendre functions. For the $r$ dependence, truncated expansions in Chebyshev polynomials are used. The equations are time-stepped by treating all nonlinear terms explicitly with a second-order Adams-Bashforth scheme, whereas all linear terms are included in an implicit Crank-Nicolson step.

In addition, we specify the fundamental (lowest nonzero) azimuthal number $m_{0}$ as a computational parameter. Thus, only the following azimuthal harmonics are really considered:

$$
1, \mathrm{e}^{ \pm \mathrm{i} m_{0} \varphi}, \mathrm{e}^{ \pm 2 \mathrm{i} m_{0} \varphi}, \mathrm{e}^{ \pm 3 \mathrm{i} m_{0} \varphi} \ldots
$$

In other words, we impose an $m_{0}$-fold symmetry in the $\varphi$ direction. If $m_{0} \neq 1$, this reduces the computation time.

\section{Results}

We compare here two cases, which differ in the shell thickness by a factor of eight. Accordingly, the sizes of the convection cells obtained in computations also differ greatly. In the language of solar physics, the cells in the case of the "thick" shell resemble and correspond in their size to the largest known cells of solar convection - the giant cells, which fill the entire thickness of the convection zone. If computations are carried out for the "thin" shell, the cells appear similar to those localized in a subsurface layer of the Sun and can be associated with the supergranules.

\section{1. "Thick" shell}

First, we describe the case of a "thick" shell with internal heating, for which we assumed $\eta=0.6, R_{\mathrm{i}}=3000, R_{\mathrm{e}}=-6000, \tau=10, P=1, P_{\mathrm{m}}=30$, and $m_{0}=5$. A quadratic term was present in the temperature dependence of density. The distributions of the 

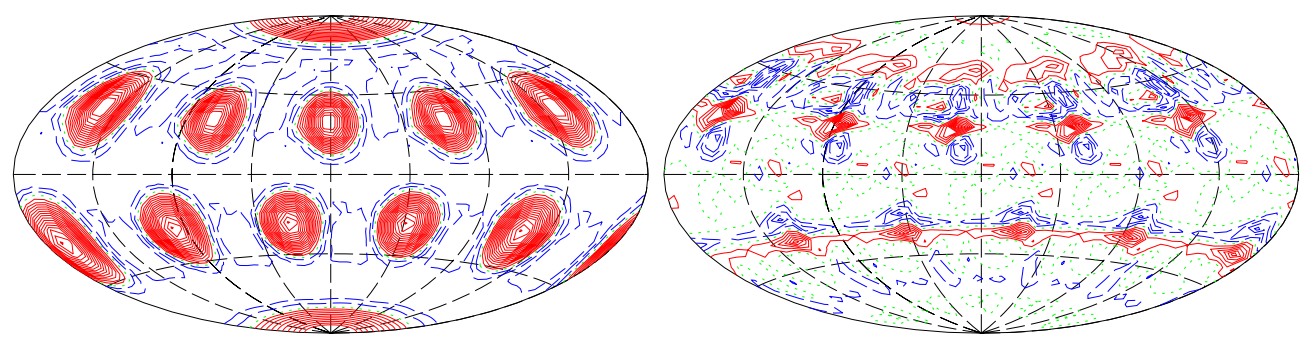

Figure 2. Typical patterns of the radial velocity component on the sphere $r=r_{\mathrm{i}}+0.5$ (left) and of the radial magnetic-field component on the sphere $r=r_{\mathrm{o}}$ (right) in the thick-shell model. Positive values are represented by solid (red) contours, zero values by dotted (green) contours, and negative values by dashed (blue) contours.

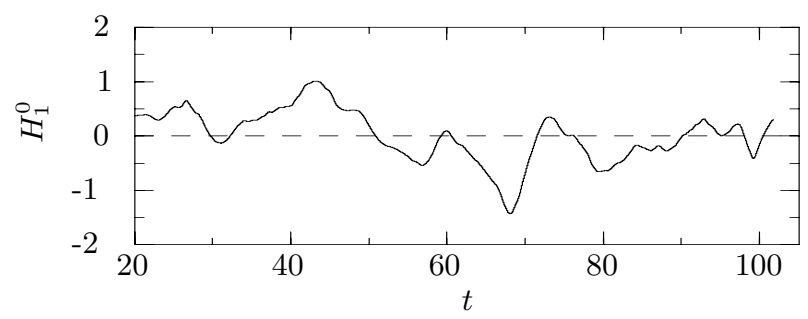

Figure 3. Variation in the amplitude of the dipolar harmonic of the poloidal magnetic field, $H_{1}^{0}(r, t)$, at $r=r_{\mathrm{i}}+0.5$ in the thick-shell model.

temperature $T_{\mathrm{S}}(r)$ and its gradient $\mathrm{d} T_{\mathrm{S}} / \mathrm{d} r$ for the corresponding static-equilibrium state are shown in figure 1. Obviously, the outer part of the shell is convectively unstable and the inner part is stable.

In this case, a very stable pattern of convection cells with a dodecahedral symmetry can be observed (figure 2), and it is especially "stiff" because of the fivefold symmetry imposed. The entire pattern drifts in the retrograde direction, in agreement with theoretical predictions (Busse 2004).

It is interesting that the pattern of differential rotation obtained in our simulations resembles the pattern actually observed on the Sun, although no anisotropic turbulence is included in the model (this suggests that convection affected by rotation, even on large scales, is in a sense similar to such turbulence).

The pattern of magnetic field is more involved than the flow. However, we have to note some remarkable features of the computed dynamo process, which is cyclic, although not quite regular.

First, local magnetic structures associated with convection cells emerge repeatedly as bipolar magnetic regions (see figure 2). They change their configuration and finally dissipate into much weaker remnant fields. In general outline, the pattern of bipolar regions resembles the pattern of solar magnetic bipoles.

Second, the dipolar component of the global magnetic field exhibits polarity reversals (see figure 3 for a graph of the amplitude of the dipole component, $H_{1}^{0}$ ). The background fields - remnants of the decaying local magnetic structures — drift toward the poles and "expel" the "old" background fields present in the polar regions. Such poleward drift of the new polarity, which replaces the old one, also resembles the process actually observed on the Sun. The resulting polarity reversal can be directly observed if we follow the magnetic-field pattern in the polar regions. 

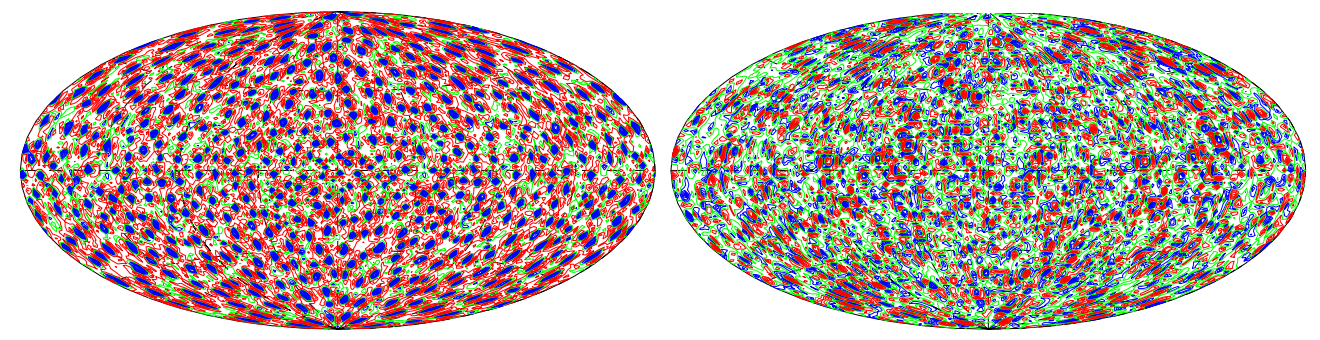

Figure 4. Typical patterns of the radial velocity component on the sphere $r=r_{\mathrm{i}}+0.5$ (left) and of the radial magnetic-field component on the sphere $r=r_{\mathrm{o}}$ (right) in the thin-shell model. The same form of representation as in figure 2 is used.

Third, an interesting intermittent behaviour is exhibited by the variations in the full magnetic energy of the system and in two particular fractions of the energy associated with the axisymmetric and nonaxisymmetric parts of the dipolar components of the magnetic field (i.e., those that are antisymmetric with respect to the equatorial plane). The axisymmetric part is represented by the spherical harmonics with $l$ odd and $m=0$ [see (2.5)], and the nonaxisymmetric part by other harmonics with $l+m$ odd. As can be seen from the corresponding diagram (not presented here), the main peaks in the graph of the total energy are alternately associated with increases in the energies of the axisymmetric and the nonaxisymmetric part of the magnetic-field component with a dipolar symmetry. To our knowledge, no counterpart to this phenomenon has been revealed on the Sun.

\section{2. "Thin" shell}

Another cellular-dynamo regime corresponds to the case of a "thin" shell, for which $\eta=0.95$. Internal heating is again specified, with $R_{\mathrm{i}}=3$ and $R_{\mathrm{e}}=0$, and a quadratic term in the temperature dependence of density is also specified. The other parameters are $P=P_{\mathrm{m}}=1, \tau=10$, and $m_{0}=2$. The static temperature profile has a very small curvature in this case, being visually indistinguishable from a linear, conductive profile.

Typical patterns of the velocity field and magnetic field observed in this case are shown in figure 4. In this case, the "background" fields cannot be separated from the fields of "active regions" (which pack the spherical surface very closely), and the evolution of the fields reaches shortly a nearly stationary regime. No polarity reversals are observed, and the energy varies little.

\section{Discussion and conclusion}

Thus, we have constructed relatively simple numerical models of the self-sustained process of generation of interacting global and local magnetic fields. As in the hypothetical solar dynamo, the generation is driven by thermal convection in combination with differential rotation. Let us make some remarks concerning the possibility of reproducing, in this way, the patterns actually observed on the Sun.

On the one hand, the "dodecahedral" pattern of large cells is highly stable and does not undergo any changes other than the overall azimuthal drift. For this reason, the sites of local amplification and structuring of the magnetic field are fixed to certain latitudes. Therefore, the latitudinal drift of the generation zones of local fields is prohibited under the conditions of the thick-shell model, and even if the model includes many important dynamical properties of solar convection, it nevertheless will not reproduce such features as, e.g., the butterfly diagram. The global magnetic field is, however, developed fairly 
well in this model and exhibits irregular variations, which include redistribution over latitudes and sign reversals.

On the other hand, the thin-shell model clearly demonstrates the possibility of the above-mentioned local processes on a much smaller scale. It might be speculated that, if the large-scale seed field from which local fields originate should drift latitudinally and exhibit cyclic variations, the pattern of local fields would visually exhibit these changes. In our thin-shell model, however, the pattern of convection cells and local fields is fairly uniform and the global field varies little, as can be judged by the slow and smooth variations in the amplitudes of the lowest harmonics and in the energy associated with the global magnetic field.

Thus, a step toward more realistic simulations of solar-type dynamos could likely be made if at least two characteristic scales of convection are reproduced in the model, e.g., those present in the two models described here. In this case, the interaction of small-scale cells with the large-scale magnetic field would appear as the control of the generation of local magnetic fields by the global field.

\section{References}

Busse, F.H. 2004, Chaos 14, 803

Dobler, W. \& Getling, A.V. 2004, in: A.V. Stepanov, E.E. Benevolenskaya, \& A.G. Kosovichev (eds.), Multi-Wavelength Investigations of Solar Activity, Proc. IAU Symp. No. 223, St Petersburg, 14-19 June 2004 (Cambridge University Press), p. 239.

Getling, A.V. \& Tverskoy, B.A. 1971a, Geomagn. Aeron. 11, 211

Getling, A.V. \& Tverskoy, B.A. 1971b, Geomagn. Aeron. 11, 389

Getling, A.V. 2001, AZh 78, 661 (Engl. transl: Astron. Rep. 45, 569)

Tilgner, A. 1999 Int. J. Num. Meth. in Fluids 30, 713

Tilgner, A. \& Busse, F.H. 1997, J. Fluid Mech. 332, 359

Tverskoy, B. A. 1966, Geomagn. Aeron. 6, 11.

\section{Discussion}

BRANDENBURG: What fraction of the total magnetic energy is contained in the dipolar component?

Gething: Typically, the energy associated with the axisymmetric part of the dipolar component of the magnetic field ranges from one-fifth to one-third of the total magnetic energy. The largest values of this fraction are reached at the energy maxima.

Tikhomolov: Did you include rotation? How does it influence the patterns in magnetic field and convection cells?

GETLING: Rotation is here an essential ingredient of the dynamo mechanism. It should, however, be kept in mind that the rotation of the whole pattern, which is so pronounced in the movie illustrating the case of the thick shell, is a retrograde drift of the pattern rather than the rotation of the system itself. The latter was subtracted, so that the movie represents the pattern in the frame of reference rotating with the system. 